\title{
A Persistent Left-Sided Superior Vena Cava With Agenesis of the Right Superior Vena Cava in a Patient Who Underwent Liver Transplantation: A Case Report
}

\author{
Charalampos Lalenis ${ }^{\mathrm{a}, \mathrm{c}}$, Alice Pittaro ${ }^{\mathrm{a}}$, Federico Geraldini ${ }^{\mathrm{b}}$, Giulio Barbiero ${ }^{\mathrm{a}}$, Annalisa Boscolo ${ }^{\mathrm{b}}$, \\ Diego Miotto ${ }^{\mathrm{a}}$
}

\begin{abstract}
The persistent left-sided superior vena cava (PLS-SVC) is the most frequent congenital anomaly of the mediastinal veins system. The concomitant agenesis of the right-sided superior vena cava (RS-SVC) is much less frequent. This variant is often discovered incidentally and could be mistaken for other findings or complicated intravascular accesses. A 29-year-old Caucasian woman presented with a decompensated autoimmune cirrhosis and hyperbilirubinemia. After a successful liver transplantation, a central venous catheter (CVC) was placed through the right jugular and a chest X-ray examination demonstrated its position into the left subclavian vein. After some days, it has been replaced by another CVC positioned from the left jugular vein. Using ultrasonography during the positioning maneuvers, we observed the metallic guide of the CVC in what seemed to be the dilated coronary sinus (CS). Subsequent chest X-ray examination demonstrated the catheter's tip in the left paramediastinal position. In order to confirm the catheter's exact position, we chose to inject contrast medium through the CVC under fluoroscopic guidance; the examination confirmed the presence of a PLS-SVC. These findings were also visible in a computed tomography $(\mathrm{CT})$ examination previously performed in another hospital, which demonstrated the agenesis of the RS-SVC as well. We demonstrated a rare case of young patient with a PLS-SVC and agenesis of RS-SVC who underwent major surgery (liver transplantation) without any intra- or post-operative complications, even if advanced venous accesses with high flow catheters were used.
\end{abstract}

Keywords: Persistent vena cava; Anomaly; Catheter; Liver; Transplantation; PLS-SVC; RS-SVC; Agenesis

\footnotetext{
Manuscript accepted for publication April 18, 2016

${ }^{a}$ University Radiology, Department of Medicine, University Hospital of Padua, 2 Via Giustiniani, 35128 Padua, PD, Italy

${ }^{\mathrm{b}}$ Anesthesiology, Department of Medicine, University Hospital of Padua, 2 Via Giustiniani, 35128 Padua, PD, Italy

${ }^{\mathrm{c} C o r r e s p o n d i n g ~ A u t h o r: ~ C h a r a l a m p o s ~ L a l e n i s, ~ V i a ~ C o r t u s i o ~ 2, ~} 35128$ Padova, Italy.Email: babis.lalenis@gmail.com
}

doi: http://dx.doi.org/10.14740/jmc2487w

\section{Introduction}

Persistent left-sided superior vena cava (PLS-SVC) is the most common venous mediastinal variant with a prevalence estimated approximately of $0.3-0.5 \%$ in the general population [1, $2]$ and of $4 \%$ in patients with congenital heart diseases [3]. In $90 \%$ of cases, the superior vena cava (SVC) is bilateral. Agenesis of right-sided SVC (RS-SVC) is uncommon (10-17\% of cases with PLS-SVC). PLS-SVC typically (in $90 \%$ of cases) drains into the right atrium via coronary sinus (CS) which is easily recognized as dilated by echocardiography. Alternatively, it can drain into the inferior vena cava, a hepatic vein or into the left atrium directly, via CS or through the left superior pulmonary vein. Left atrial drainage and the absence of RSVC are more frequently associated with congenital heart diseases.

\section{Embryology}

During embryonic life, the cardinal veins represent the main venous drainage system of the embryo. The cephalic part of the embryo is drained by the two anterior cardinal veins and the rest of the embryo is drained by the posterior cardinal veins. Before entering the sinus horn and, subsequently, the embryonic heart, the anterior and posterior veins unite to form the short right and left common cardinal veins. During the eighth week of gestation, the anterior cardinal veins anastomose allowing blood to drain from the left anterior cardinal vein into the right anterior cardinal vein. This anastomosis grows and ultimately forms the left brachiocephalic (innominate) vein. The part of the left anterior cardinal vein distal to the brachiocephalic anastomosis becomes the "left internal jugular vein" and unites with the "left subclavian vein" from the developing upper limb. The caudal portion of right superior cardinal vein forms the normal RS-SVC. The part of the left anterior cardinal vein proximal to the anastomosis normally regresses/ atrophies to become "the ligament of Marshall" (Fig. 1b). Failure of this normal regression leads to a persistent left-sided vascular structure (the PLS-SVC) that, in most cases, empties into the CS. The innominate vein may or may not degenerate, so a bridging innominate vein may or may not be present. An absence of the innominate vein associated with PLS-SVC oc- 


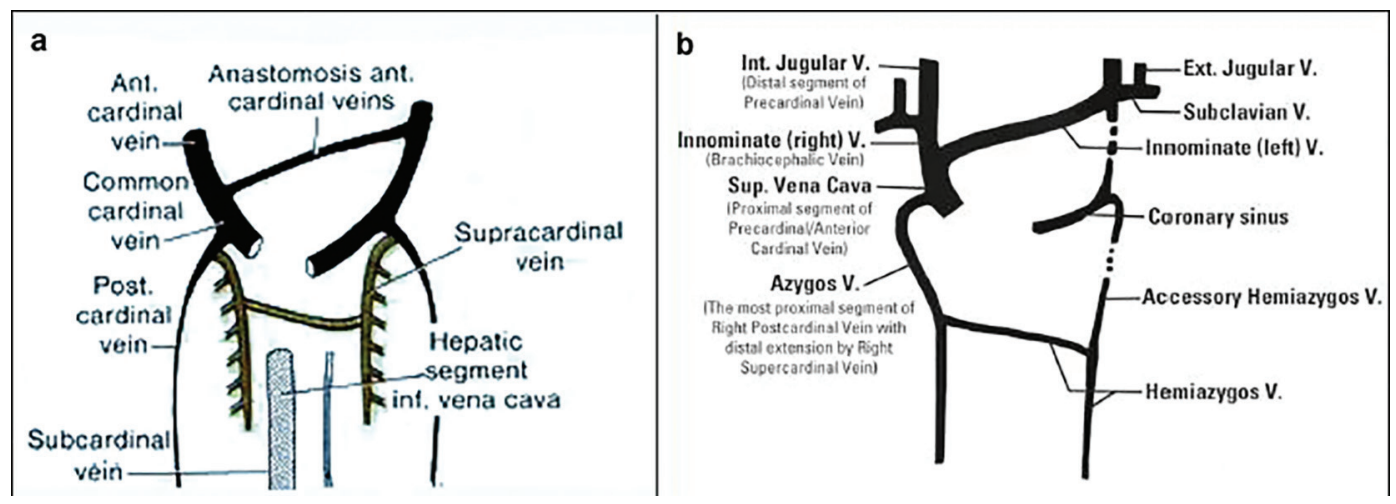

Figure 1. Precardinal/anterior cardinal vein developmental process (image taken from Phlebolymphology. Vol. 19, No. 4, 2012).

curs in $65 \%$ of cases.

The insertion of the PLS-SVC is also variable. In $90 \%$ of cases, the PLS-SVC drains into the right atrium via the CS without any hemodynamic consequence. In the rest $10 \%$ of individuals, it may drain into the left atrium resulting in a slight right-to-left shunt; in that case, if other anatomical variations are associated (such as absence of the RS-SVC), the resulting shunt may be of greater amount. In case of PLS-SVC, azygos veins may be reversed: the hemiazygos vein (the remnant of the proximal part of the left posterior cardinal vein) located on the left can be hypertrophic and drain directly into the leftsided SVC, in the way that a normal azygos vein (the remnant of the proximal part of the right posterior cardinal vein) would drain into the SVC on the right side $[4,5]$.

\section{Case Report}

A 29-year-old woman was transferred to our hospital with decompensated autoimmune cirrhosis and hyperbilirubinemia (MELD 28 e Child Pugh score C12). Two weeks after hospitalization, the patient underwent liver transplantation. Before

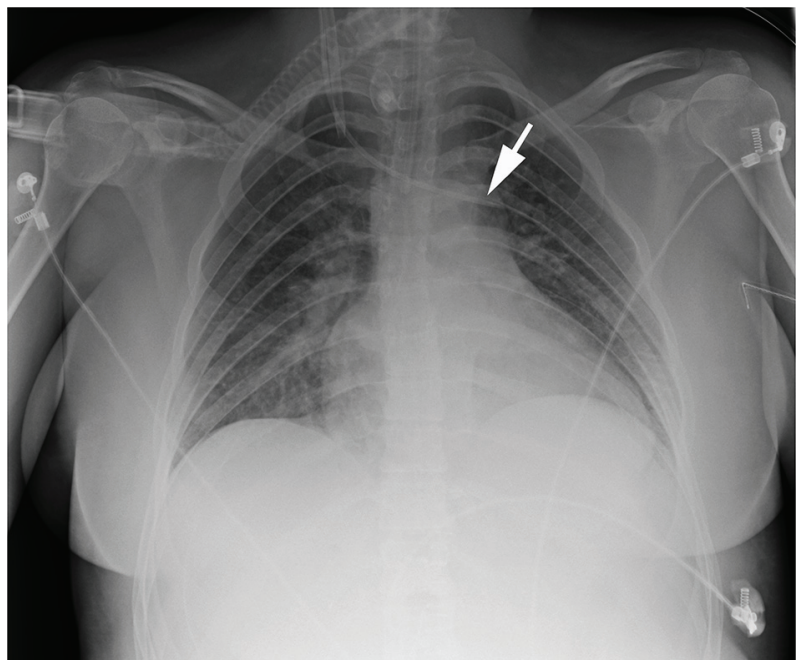

Figure 2. The white arrow demonstrates the tip of the CVC apparently in the left subclavian vein. the intervention, a central venous catheter (CVC) was placed via the right jugular vein. An antero-posterior chest X-ray obtained subsequently described the tip of the CVC (white arrow) as placed "in the left subclavian vein" (Fig. 2).

Three days after, the patient showed signs of infection at the site of access of the CVC, thus it was replaced with another one in contralateral position, via the left jugular vein. The difficulty in establishing a valid central venous access prompted further investigation. We used ultrasonographic guidance to confirm the correct position of the metallic guide of the CVC in the left jugular vein. During the positioning, we noticed that the CS (*) was dilated. Furthermore, once the catheter was inserted, its tip appeared to be directly into the CS (Fig. 3, white arrow).

A blood gas measurement directly from the $\mathrm{CVC}$ revealed normal venous $\mathrm{pCO}_{2}$ and $\mathrm{pO}_{2}$ values. Despite the unclear positioning, the CVC was placed and we decided to control its position radiographically. An antero-posterior chest X-ray obtained subsequently revealed that "A CVC was placed via the left jugular vein and its probe tip was projected over the left heart chambers probably as a result of a PLS-SVC" (Fig. 4).

The differential diagnoses of a venous catheter projected over a left paramediastinal position are the following: 1) Venous position: left-sided SVC, left internal thoracic vein, left

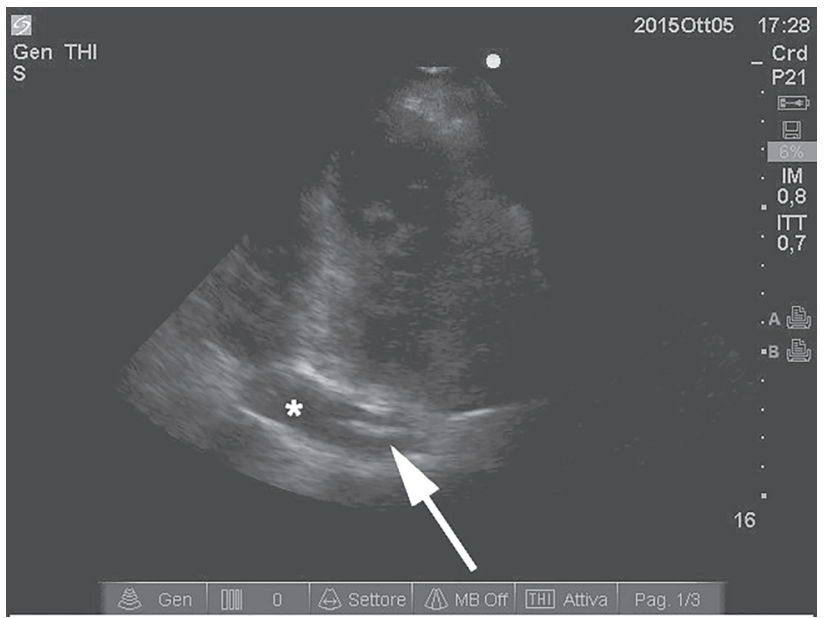

Figure 3. Ultrasonographic imaging demonstrating the metallic guide of the CVC (white arrow) in a dilated coronary sinus $\left({ }^{*}\right)$. 


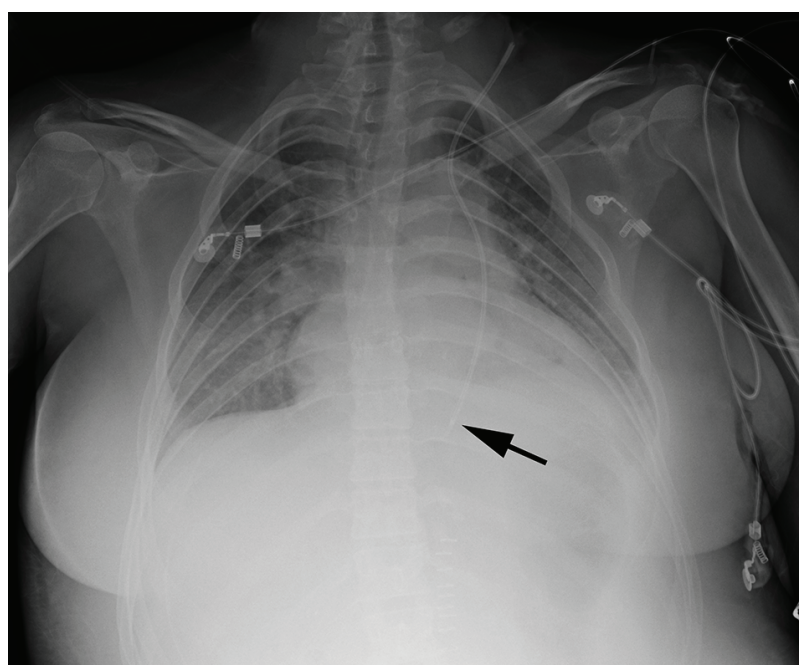

Figure 4. The black arrow demonstrates the tip of the CVC, apparently in the PLS-SVC.

superior intercostal vein, left pericardiophrenic vein. 2) Arterial position: left carotid artery to descending thoracic aorta. 3) Extravascular position: directly into the mediastinum, into the pleural space.

We decided to inject water-soluble radiographic contrast medium (Iohexol, OMNIPAQUE ${ }^{\mathrm{TM}} 350$ ) through the CVC under fluoroscopic guidance to determine in which vein the CVC was placed. Early opacization of the pulmonary artery shortly after the injection (white arrow) revealed that the probe's tip was placed in the PLS-SVC which drained into the right atrium (Fig. 5).

A closer look in the computed tomography (CT) examination previously performed in another hospital, not available until then, revealed not only a PLS-SVC, but also the absence of the RS-SVC (Fig. 6). In particular, the right innominate vein (1) drained into the PLS-SVC (3) via a bridging vein (the right brachiocephalic vein crossing the midline) (2). The PLS-SVC drained subsequently firstly into the CS (4) and then into the right atrium (5). Note the severe dilation of the CS (4) which received blood from the head and the upper limbs (Fig. 6). These findings were not mentioned in the CT report.

Another interesting finding was that the hemiazygos vein (6) on the left side was hypertrophic and drained directly into the PLS-SVC just like a normal azygos vein would do on the right side in presence of an RS-SVC (Fig. 7).

\section{Discussion}

PLS-SVC is the most common congenital thoracic venous anomaly, with a prevalence of $0.3-0.5 \%$ in the general population [6].

Commonly, the persistence of PLS-SVC results in the presence of both left and right SVCs. In some rare cases, the caudal right superior cardinal vein regresses, leading to an absent RS-SVC with PLS-SVC; if this occurs, the PLS-SVC drains all the blood from cranial part of the body.

The persistence of the left SVC may have some implications in clinical practice, warranting the need to investigate its presence when in doubt. An increased risk of complications during positioning of CVCs, Swan-Ganz catheters and pace makers in the PLS-SVC has been reported. The manipulation of a CVC in or through the dilated CS can be complicated by hypotension, angina, thrombosis and cardiac arrest [7]. There may also be a higher incidence of arrhythmias in these patients [8].

Interestingly, in this case, the patient has undergone major abdominal surgery (liver transplantation) without any complications related to the manipulation and use of a CVC in the PLS-SVC. A Swan-Ganz catheter was not positioned, but a standard CVC was positioned pre-operatively in the right jugular vein. Furthermore, a rapid infusion system (RIS) has also been used intra-operatively through an advanced venous access (AVA) high flow catheter also placed in the right jugular vein, with no adverse consequences despite the high rates of infusion.

The patient also underwent three separate attempts of positioning a valid central venous access during her stay in the intensive care unit. The attempts were all marred by complicated positioning, with chest radiography reporting progression of

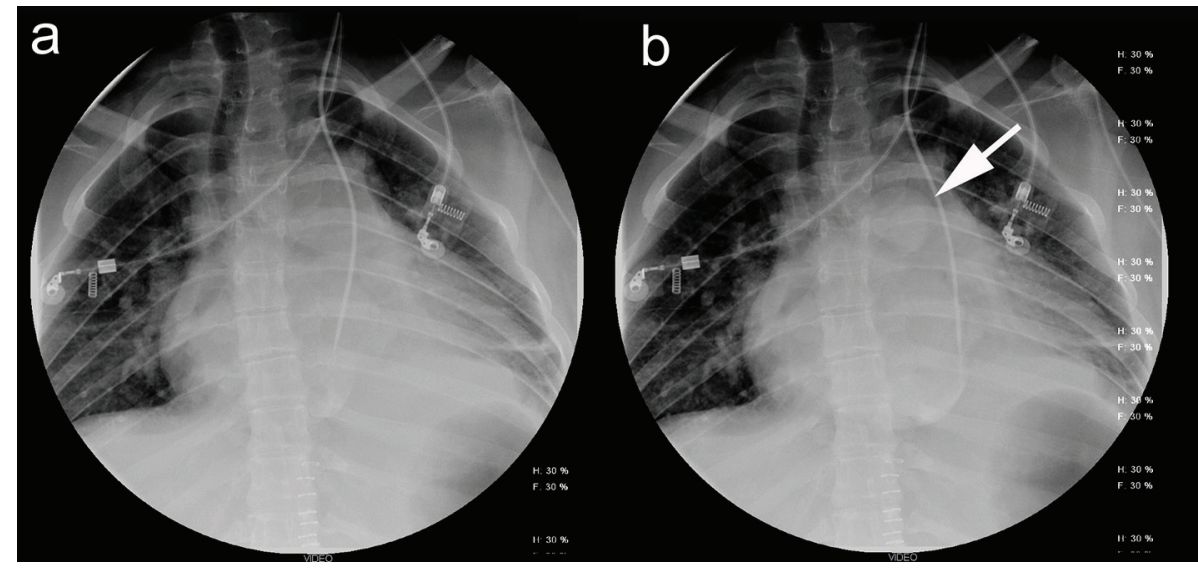

Figure 5. (a) Injection of contrast medium directly from the CVC into the dilated CS. (b) Early opacization of the pulmonary artery demonstrates that the PLS-SVC drains into the right atrium. 


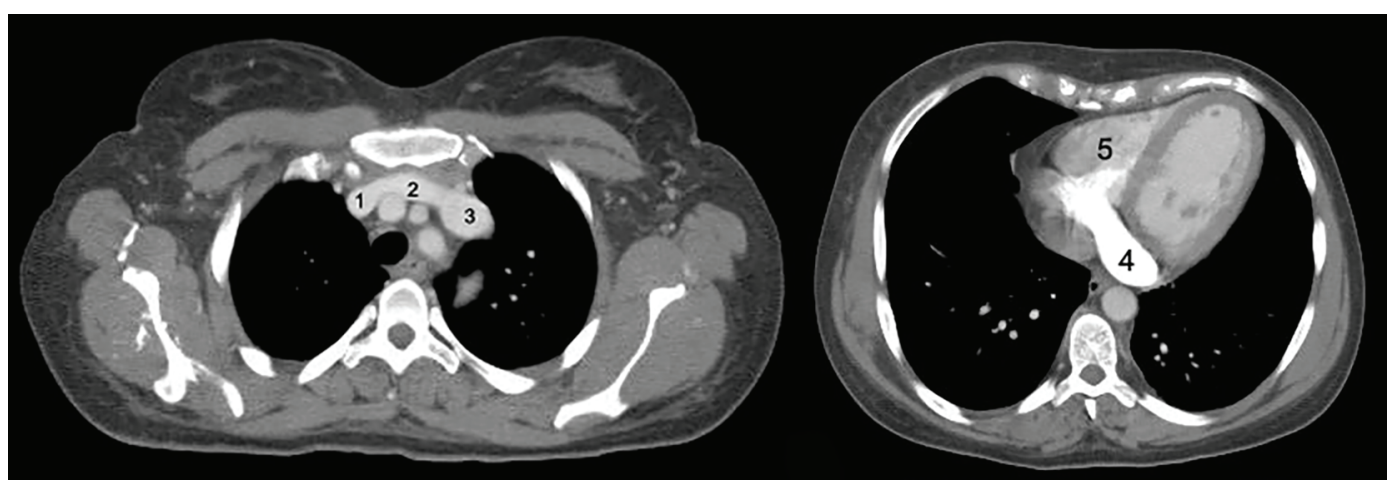

Figure 6. On the left: CT axial view of the PLS-SVC and the right brachiocephalic vein crossing the midline. On the right: the dilated coronary sinus.

the catheter from the right jugular to the left subclavian vein in the first two attempts. The third attempt was made from the contralateral side, using the left jugular vein as access point. Furthermore, ultrasonography has been used in order to confirm correct positioning. During the maneuvers, the metallic guide was observed in what appeared to be the CS, extremely dilated; despite this finding, the catheter was placed and a chest $\mathrm{X}$-ray was requested to confirm the exact position.

As with this case, diagnosis of PLS-SVC is usually an incidental finding. Positioning through the left internal jugular vein demonstrates a typical unusual course of the catheter on the chest X-ray which often prompts further investigation. Transthoracic echocardiography almost always reveals a dilated CS and the diagnosis is conventionally confirmed through the use of saline contrast echocardiography (commonly known as "bubble test"). In case of PLS-SVC, early CS opacification (before the right atrium) is observed when a saline contrast is injected in the left brachial vein. In our case, since the anteroposterior chest X-ray was not enough to evaluate the exact position of the catheter, fluoroscopy was elected to validate the ultrasonographic finding, confirming the placement of the catheter in the PLS-SVC.

\section{Conclusion}

An unusual course of the CVC on the chest X-ray associated with the presence of a dilated CS on echocardiography should alert the clinician towards the possibility of PLS-SVC. The diagnosis can be usually confirmed by saline contrast echocardiography (bubble test). The presence of a PLS-SVC certainly presents technical difficulties, but does not usually preclude insertion of catheters. The additional associated risks should be taken in consideration and demand more attention during the manipulation of the catheters. It must be noted that in this case the usual preoperative screening done in potential liver transplant receivers failed to detect this anomaly and the patient underwent a very intensive surgical procedure without an accurate analysis of the venous vasculature. Furthermore, alternative access sites should probably be considered in presence of PLS-SVC as the possibility of complications during the placement of catheters in that case is well documented. This case report emphasizes the need for accurate pre-intervention investigation in order to determine the presence of vascular anomalies, especially in cases of major surgery such as, in our case, a liver transplantation.

\section{Competing Interests}

The authors declare that they have no competing interests.

\section{Author Contributions}

SKG: collection of data, patient care and preparation of man-

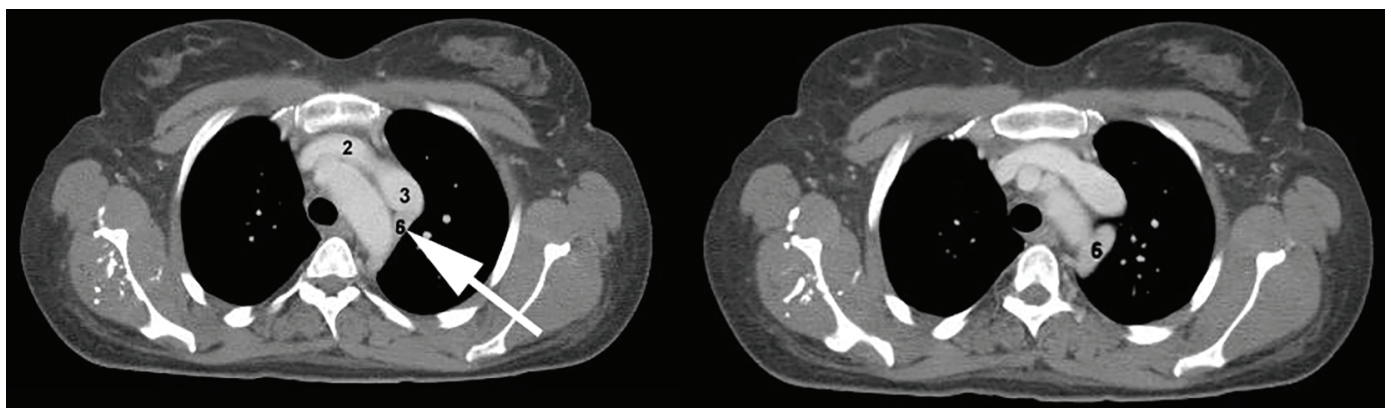

Figure 7. The white arrow indicates a hypertrophic hemiazygos vein (6) which drains directly into the PLS-SVC just like a normal azygos vein would do on the right side. 
uscript. SRP and GV: preparation of manuscript. FLR: final revision of manuscript and guidance. All authors read and approved the final manuscript.

\section{Abbreviations}

PLS-SVC: persistent left-sided superior vena cava; CS: coronary sinus; CVC: central venous catheter; CT: computed tomography; RIS: rapid infusion system; AVA: advanced venous access

\section{References}

1. Irwin RB, Greaves M, Schmitt M. Left superior vena cava: revisited. Eur Heart J Cardiovasc Imaging. 2012;13(4):284-291.

2. Goyal SK, Punnam SR, Verma G, Ruberg FL. Persistent left superior vena cava: a case report and review of litera- ture. Cardiovasc Ultrasound. 2008;6:50.

3. Cha EM, Khoury GH. Persistent left superior vena cava. Radiologic and clinical significance. Radiology. 1972;103(2):375-381.

4. Webb WR, Gamsu G, Speckman JM, Kaiser JA, Federle MP, Lipton MJ. Computed tomographic demonstration of mediastinal venous anomalies. AJR Am J Roentgenol. 1982;139(1):157-161.

5. Chuang VP, Mena CE, Hoskins PA. Congenital anomalies of the inferior vena cava. Review of embryogenesis and presentation of a simplified classification. Br J Radiol. 1974;47(556):206-213.

6. Wood P. Disesase of heart and circulation. 2. Philadelphia: JB Lippincott; 1956.

7. Albouaini K, Rao A, Ramsdale D. Pacing in Patients With Congenital Heart Disease Cardiol. 2013;20(3):117-120.

8. Morgan LG, Gardner J, Calkins J. The incidental finding of a persistent left superior vena cava: implications for primary care providers-case and review. Case Rep Med. 2015;2015:198754. 